\title{
EMBRYONIC DEVELOPMENT OF IDENTIFIED NEURONS: ORIGIN AND TRANSFORMATION OF THE H CELL ${ }^{1}$
}

\author{
COREY S. GOODMAN,*' $¥$ MICHAEL BATE,§ AND NICHOLAS C. SPITZER $\ddagger$
}

* Department of Biological Sciences, Stanford University, Stanford, California 94305; $\$$ Department of Biology, B.022, University of California, San Diego, La Jolla, California 92093; and §Max-Planck-Institut für Virusforschung, Tübingen, F. D. R.

\begin{abstract}
We describe the origin and the transformation of a single neuron, the $\mathrm{H}$ cell, which assumes two different roles during grasshopper embryogenesis. The $\mathrm{H}$ cell originates from the single cell division of midline precursor 3 (MP3). In the metathoracic (T3) segment, the $\mathrm{H}$ cell first appears as one of a pair of central pioneer fibers and later transforms into an unpaired identified neuron. During the course of its transformation, the $\mathrm{H}$ cell loses its original morphology and acquires new morphological and physiological properties. The $\mathrm{H}$ cell acquires many of the same phenotypes as the first progeny of the median neuroblast (MNB); the processes of the first MNB progeny contact the soma of the $\mathrm{H}$ cell and these cells are electrically coupled prior to the $\mathrm{H}$ cell transformation.
\end{abstract}

The differentiation of a cell often commits it to a particular fate for the remainder of its life. However, in the nervous system, individual nerve cells have been described which transform their morphology or physiology after their initial differentiation such that they lead essentially two different lives. In the nematode Caenorhabditis elegans, during post-embryonic development, individual motoneurons change both the muscles upon which they synapse and some of their presynaptic inputs (White et al., 1978). In the moth Manduca sexta, during the metamorphosis from larval caterpillar to adult moth, individual motoneurons dramatically change their morphology (Truman and Reiss, 1976). Nerve cells can also change their properties in tissue culture. Immature neurons of the rat superior cervical ganglion in vitro can change their transmitter synthesis; dual-function cells have been described that appear to represent intermediate stages in the transformation (reviewed by Patterson, 1978).

In this paper, we describe the passage of a single nerve cell through two phases of differentiation during grasshopper embryogenesis. We previously reported on the major class of neuronal precursor cells, the neuroblasts (NBs) (Bate, 1976a) and on the differentiation and cell lineage of the progeny of one of these NBs, the median neuroblast (MNB) (Goodman and Spitzer, 1979; Goodman et al., 1979b; Goodman et al., 1980; Goodman and

\footnotetext{
' This work was supported by the Helen Hay Whitney Foundation and a National Science Foundation grant to C. S. G., Deutsche Forshungsgemeinschaft to C. M. B., and National Institutes of Health and National Science Foundation grants to N. C. S. We thank E. B. Grunewald, J. Coulombe, and K. Ridge for their technical assistance and W. Kristan and A. Selverston for generous use of their facilites.
}

Spitzer, 1980a, b). Here we report on the differentiation of an identified neuron, the " $\mathrm{H}$ " cell, which is the descendant of a different class of precursors, the midline precursor cells (MPs) (Bate and Grunewald, 1980).

The embryonic development of the $\mathrm{H}$ cell in the metathoracic (T3) segment includes a major transformation which involves the loss of its initial morphology and the acquisition of new morphological and physiological properties. The $\mathbf{H}$ cell originates from the single cell division of midline precursor 3 (MP3). It first appears as one of a pair of central pioneer fibers (i.e., among the first cells to extend processes and establish the early fiber pathways) and then transforms into an unpaired identified neuron. Its sibling often dies, although when it persists, it retains only its original form.

In the following paper (Bate et al., 1981), we describe the segmental differences in the survival and cell death of the two progeny of MP3. Furthermore, we show that in those segments in which these cells survive, they develop segment-specific differences in their morphology and physiology. Some of these results have previously been reported (Goodman et al., 1979a; Spitzer et al., 1979).

\section{Materials and Methods}

Embryos of the grasshopper Schistocerca nitens were obtained from a laboratory colony. Embryonic development takes 20 days at $35^{\circ} \mathrm{C}$ (Bentley et al., 1979). In this study, we used four methods, all of which have been previously described: (i) reconstructions of fixed cells from $1-\mu \mathrm{m}$ plastic sections for light microscopy and ultrathin sections for electron microscopy (Bate, 1976a, b; Bate and Grunewald, 1980); (ii) direct observation of living cells with Nomarski interference contrast optics 
(Goodman and Spitzer, 1979; Goodman et al., 1979b); (iii) intracellular staining by injection of the fluorescent dye Lucifer Yellow (Goodman and Spitzer, 1979; Goodman et al., 1979b); and (iv) intracellular stimulation and recording by microelectrode penetration of cell bodies (Goodman and Spitzer, 1979; 1980a, b).

\section{Results}

\section{The neuroblasts}

The grasshopper is a segmental animal whose central nervous system (CNS) consists of a brain or supraesophageal ganglion (arising from several fused embryonic segments), a subesophageal ganglion (arising from three fused embryonic segments), and a chain of segmental ganglia running along the ventral surface of the three thoracic and 11 abdominal segments. There are three thoracic ganglia: the prothoracic (T1), the mesothoracic (T2), and the metathoracic (T3) ganglion. During embryogenesis, the T3 ganglion fuses with the first three abdominal ganglia (A1 to A3). The next four abdominal ganglia (A4 to A7) remain separate, and the last four abdominal ganglia (A8 to A11) fuse during embryogenesis to form the terminal abdominal ganglion. In this and the following paper, we examine eight of the segmental ganglia: T2, T3, and A1 to A6. In the adult, each thoracic ganglion contains $\sim 3000$ neurons and each abdominal ganglion $\sim 500$ neurons.

There are two distinctly different classes of precursor cells in grasshopper embryos that give rise to the neurons of the segmental ganglia: the neuroblasts and the midline precursor cells. On the ventral surface of each body segment of a 5-day embryo are the paired plates of neuroblasts (NBs) (Fig. 1, $A$ and $B$ ), the major class of neuronal precursor cells. The NBs, enlarged ectodermal cells, were first described by Wheeler (1893) and recently

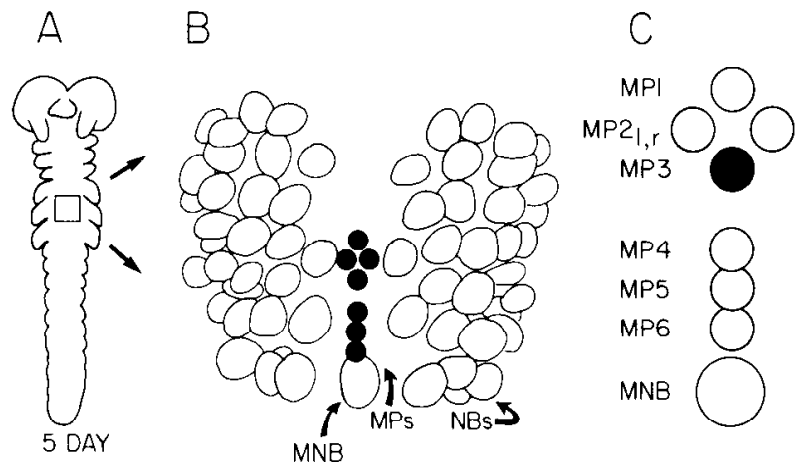

Figure 1. The location of the neuronal precursor cells in the mesothoracic (T2) segment of a day 5 grasshopper embryo (Schistocerca nitens, embryogenesis takes 20 days at $35^{\circ} \mathrm{C}$ ). Each thoracic and abdominal ganglion begins with a reiterated pattern of two different classes of precursors: neuroblasts (NBs) and midline precursors (MPs). $A$, Profile of a day 5 embryo viewed from the ventral surface, indicating the position of mesothoracic precursors (square). $B$, Camera lucida drawing of the segment showing the 30 ventral neuroblasts (NBs) on each side, the median neuroblast (MNB), and the seven midline precursors (MPs; black). C, Schematic diagram of the position and identities of the seven MPs relative to the MNB. MP3 (black) is the focus of this study. in greater detail by Bate (1976a). There are $30 \mathrm{NBs}$ in each left or right plate per segment and a single unpaired $\mathrm{NB}$, called the median neuroblast (MNB), at the posterior end of each segment. This segmental pattern of 61 NBs is constant from embryo to embryo and is precisely repeated in each of the eight segments (T2, T3, A1 to A6) that we have examined.

Each NB is a stem cell which will not itself become a neuron. Rather, each NB maintains its large size while giving rise to a chain of progeny which themselves each divide once more and differentiate into neurons. In the T3 ganglion, each NB gives rise to 10 to 100 progeny. The NBs eventually degenerate at precise times during embryogenesis. Most of the neurons in the segmental ganglia are the progeny of the NBs. Our previous studies have described the MNB and the cell lineage and differentiation of its $\sim 100$ progeny in the T3 ganglion (Goodman and Spitzer, 1979; Goodman et al., 1979b; Goodman et al. 1980). All of the progeny of the MNB are encased in a common glial sheath; this packet of neuronal somata is located along the midline of the dorsal surface of the developing T3 ganglion. The first two cell divisions of the MNB give rise to the identified neurons: DUM 3, 4, 5; DUM 4, 5; and DUM 5.

\section{The midline precursors}

Each of the thoracic and abdominal segments contains a second class of precursors in addition to the 61 NBs. There are seven cells, celled midline precursors (MPs) (Bate and Grunewald, 1980), arranged in a constant pattern on the dorsal surface just anterior to the MNB (Figs. 1 and 2). Each MP divides only once to give rise to two progeny (Fig. 2), in contrast to the $\sim 100$ progeny of the MNB. The first MP cells finish their single cell division early in embryogenesis and the other cells follow in sequence. The 14 progeny of the seven MPs send out processes that either are the first or among the earliest processes to establish the initial fiber pathways of the CNS (Bate and Grunewald, 1980). The axons of the later neuronal progeny of the NBs often appear to follow these initial pathways.

$M P 1, M P 2_{\mathrm{L}}$, and $M P 2_{\mathrm{K}}$. The processes of the progeny of MP1, MP2 $2_{\mathrm{I}}$, and $\mathrm{MP} 2_{\mathrm{R}}$ have been studied in great detail (Bate and Grunewald, 1980). The progeny of MP2 and $M P 2_{R}$ establish the two longitudinal fiber tracts (LFTs) (Fig. 2A). The progeny of MP1 also add early processes to the longitudinal fiber tracts.

$M P 3$. The progeny of MP3 also add early processes to the longitudinal fiber tracts. They are discussed in detail in the next section.

$M P 4, M P 5$, and MP6. We have examined the processes of the progeny of MP4 and MP6 by intracellular injection of the fluorescent dye Lucifer Yellow. The progeny of MP4, MP5, and MP6 are arranged in a group with the MP4 progeny the most anterior and dorsal, and the MP6 progeny the most posterior and ventral (Fig. 2). The processes of the MP4 progeny extend anteriorly and establish the dorsal median fiber tract (MFT) (Figs. 2A and 3). Just before the anterior commissure (AC), their processes bifurcate and then extend laterally in the anterior commissure. Although among the earliest processes in the anterior commissure, they are not the first; 

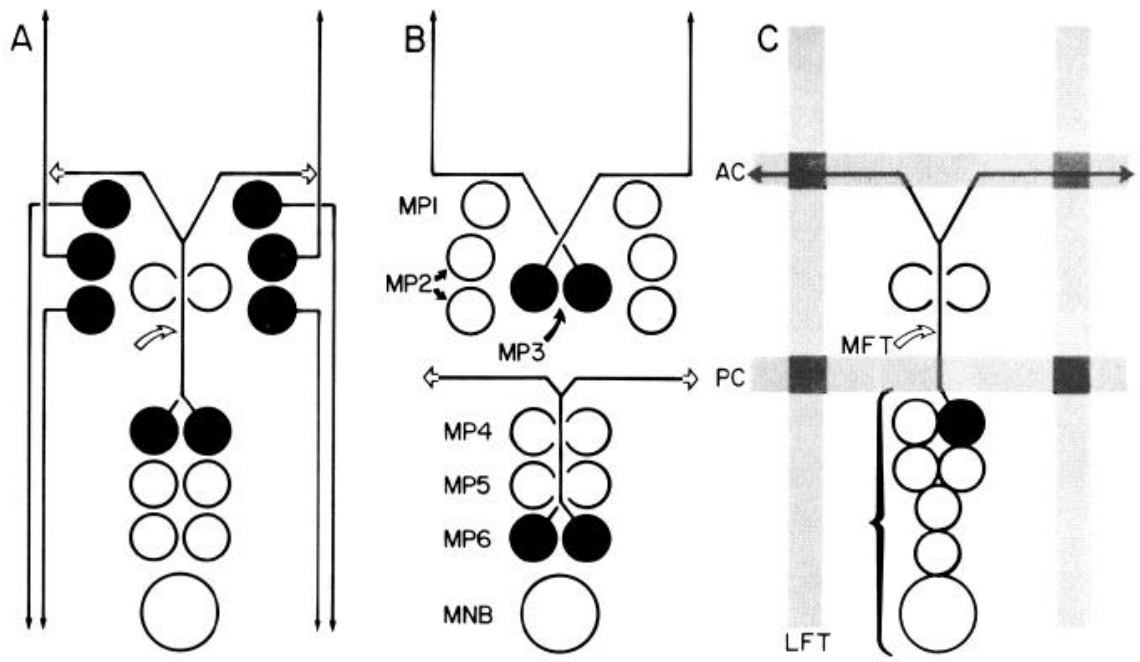

Figure 2. Schematic diagrams of the position and processes of the 14 progeny of the seven midline precursors (MPs) and the early progeny of the median neuroblast (MNB). $A$, The processes of the progeny of MP1, MP2 $2_{\mathrm{L}}$, and MP2 $2_{\mathrm{R}}$ establish the longitudinal fiber tracts (LFT). The processes of the MP4 progeny establish the median fiber tract (MFT) and bifurcate before the anterior commissure (AC). B, The MP3 progeny decussate and send processes out the contralateral sides of the AC and then anteriorly in the LFT. The MP6 progeny extend processes anteriorly that bifurcate in posterior commissure (PC). The terminations of the processes of the MP4 and MP6 progeny are presently unknown (open arrows). $C$, The early progeny of the MNB (e.g., black cell) extend processes that follow the MFT established by the MP4 progeny.

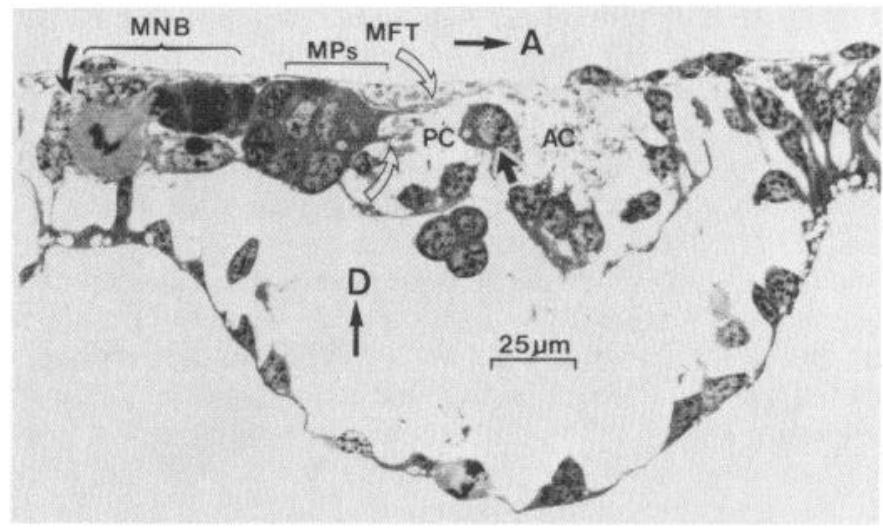

Figure 3. Photomicrograph of a sagittal midline section through the metathoracic segment of a day 7 to 8 grasshopper embryo. $A$, anterior; $D$, dorsal. The median neuroblast (MNB) and its early progeny are posterior (and dorsal, not shown in this section) to the progeny of midline precursors (MPs) 4, 5 , and 6 . The dorsal median fiber tract (MFT) has been established by the MP4 progeny, and the ventral median fiber tract (open arrow) by the MP6 progeny. One of the two MP3 progeny is seen (solid arrow) between the anterior (AC) and posterior commissures (PC). Note the MNB is in telophase.

this commissure is first established by the processes of cells situated laterally. The dorsal median fiber tract established by the MP4 progeny serves as the pathway taken by the processes of the early (and many of the later) progeny of the MNB (Fig. $2 C$ ). The processes of the MP6 progeny extend anteriorly and establish a second and shorter median fiber tract; this tract is ventral to the tract established by the MP4 progeny (Fig. 3). These processes bifurcate and extend laterally in the posterior commissure (PC) and are among the earliest fibers in it (Fig. 2). This second median fiber tract is followed by the processes of some of the later progeny of the MNB (not illustrated). The processes of the MP5 progeny also extend anteriorly; we are uncertain, however, of their pathway.

The progeny of the MPs are considered to have an embryonic role as central pioneer fibers (Bate and Grunewald, 1980). They are either the first or among the earliest processes to establish the initial fiber pathways of the CNS. They contribute to the establishment of the reiterated segmental pattern: the left and right longitudinal fiber tracts, the anterior commissure, the posterior commissure, and the median fiber tracts. Some of the MP progeny appear destined to die after their initial role as central pioneer fibers. In some of the abdominal segments, the progeny of MP3 die about half way through embryogenesis (described in detail in the following paper), long after the many axons of the neuronal progeny of NBs have greatly enlarged the axon pathways and rapidly developing neuropil. Similarly, it appears that the progeny of MP4, MP5, and MP6 also die in some of the abdominal segments (it is more difficult to follow the progeny of MP1, MP2 $2_{L}$, and $M P 2_{R}$ during later embryogenesis and thus, we do not know yet if they live or die).

The MP progeny in many respects appear analogous to the peripheral pioneer fibers, first described in the grasshopper by Bate (1976a) and more recently by Keshishian (1980). In the limb bud of the T3 segment, a single ectodermal cell appears at the tip. This precursor cell divides only once and its two progeny establish the first fiber pathways from the limb bud to the CNS, a pathway followed later in embryogenesis by the axons of the peripherally derived sensory neurons (Bate, 1978). Experiments with apparently homologous cells in crickets indicate that the axons of such peripheral pioneer cells do indeed have a guide function during axonal growth in the peripheral nervous system (Edwards et al., 1979). 


\section{The progeny of $M P 3$}

In this and the following paper, we examine in detail the two progeny of MP3. The MP3 cell lies on the dorsal surface at the very center of the developing segmental fiber pathways (Figs. 2, 4, 5, and 6). The plane of division of MP3 is often oblique (Fig. $4 C$ ), but the two cells rapidly acquire positions on the left and right of the midline. One of the two cells usually is slightly dorsal to the other. The two MP3 progeny come to be surrounded by the left and right longitudinal fiber tracts and the anterior and posterior commissures (Figs. 2, 5, and 6). Each of the MP3 progeny has a single contralateral process. The first process of each cell decussates just posterior to the anterior commissure, extends laterally in this commissure, and then extends anteriorly as one of the early processes in the developing longitudinal fiber tracts.

The processes of the MP4 progeny, described above, extend in the median fiber tract just dorsal to the somata of the MP3 progeny. The processes of the MP4 progeny here appear to contact the more dorsal of the two somata of the MP3 progeny (Fig. 7). The MP4 progeny become
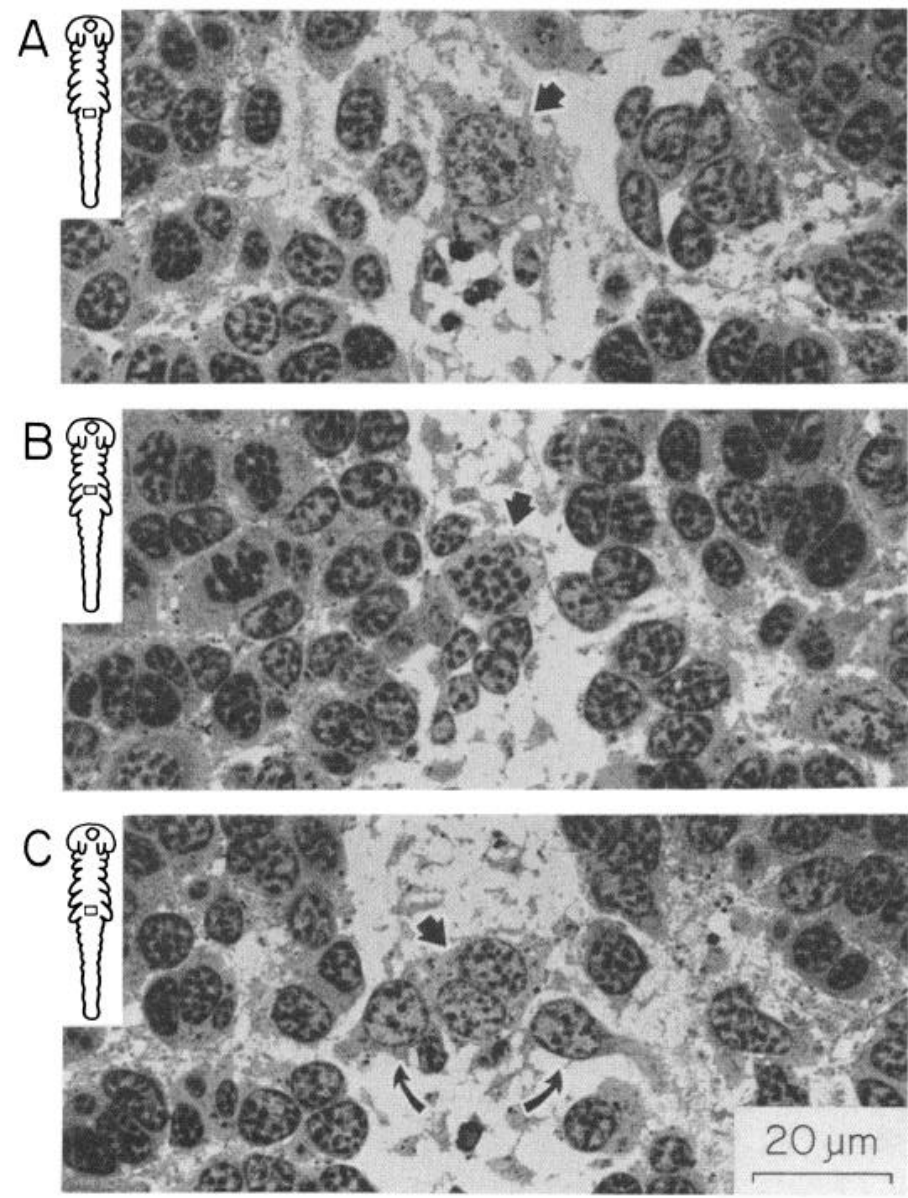

Figure 4. Photomicrographs of horizontal sections through the first abdominal $(A)$, mesothoracic $(B)$, and metathoracic $(C)$ segments showing three stages in the development of the MP3 progeny (large arrows) in a day 6 embryo. $A$, Mature MP3 cell. $B$, MP3 cell in telophase of mitosis. $C$, Two progeny of MP3. Small arrows indicate one of the two progeny each of $\mathrm{MP} 2_{\mathrm{L}}$ and $\mathrm{MP} 2_{\mathrm{R}}$.

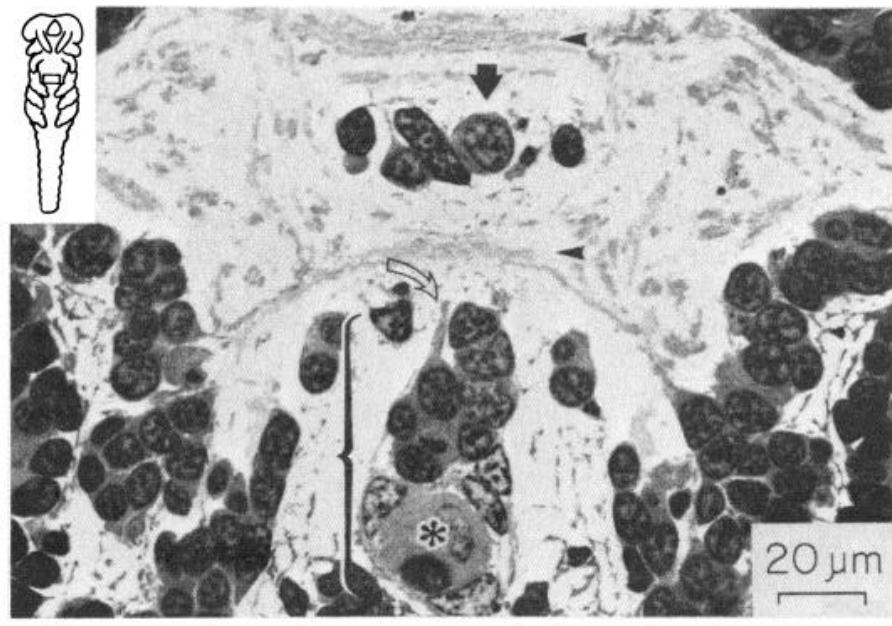

Figure 5. Photomicrograph of a horizontal section through the prothoracic segment of a day 8 embryo. The median neuroblast (*) and some of its progeny are indicated by the bracket; processes are seen extending anteriorly in the median fiber tract (open arrow). The posterior and anterior commissures are indicated (carets). The more dorsal of the two MP3 progeny (solid arrow) is seen in this section between the longitudinal fiber tracts and the two commissures.

electrically and dye coupled to the MP3 progeny. The processes of the MP3 progeny decussate at the same point where the processes of the MP4 progeny bifurcated a short time before. The axons of the early progeny of the MNB follow the same dorsal median fiber tract established by the MP4 progeny and contact the more dorsal of the somata of the MP3 progeny (Figs. $2 C, 3,6$, and 7). The axons of the MNB progeny extend over the somata of the MP3 progeny and their axons bifurcate at the same point just posterior to the anterior commissure, following the pathway established by the processes $\mathrm{C}$. the MP4 progeny.

\section{Morphological transformation of the $\mathrm{H}$ cell in the T3 segment}

One of the two progeny of MP3 in the T3 segment undergoes a transformation and it, and often its sibling (sib), survives. This secondary differentiation begins several days after its initial differentiation. The morphological transformation is illustrated by a series of drawings (Fig. 8) and photographs (Fig. 9) of cells injected with Lucifer Yellow. Either one of the two cells can undergo the transformation, but only one of them does it; the sib remains unchanged. In the cases that we have observed thus far $(n>10)$, it is always the more dorsal of the two cells that transforms, regardless of its left or right position. On day 7, the somata of the two MP3 progeny are the same size (Fig. $4 \mathrm{C}$ ); by the time the transformation begins, the soma of the transforming cell has begun to enlarge relative to its sib. The transformation begins on day 8 with the appearance of a new growth cone which extends posteriorly in the dorsal median fiber tract. Between days 8 and 10, the process bifurcates at the posterior commissure, extends laterally in the posterior commissure, and each process bifurcates again in each of the longitudinal fiber tracts. The processes then extend in both longitudinal fiber tracts to the brain and to the 

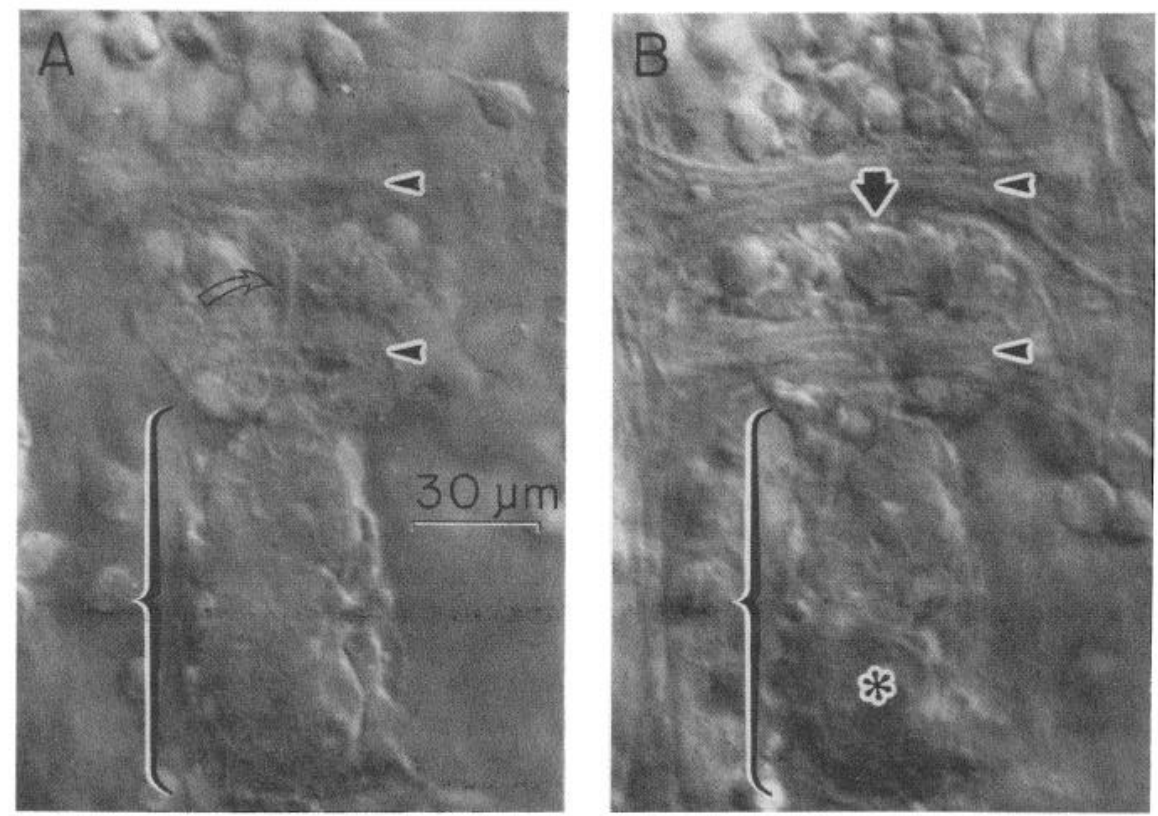

Figure 6. Photomicrographs of the dorsal aspect of the metathoracic segment of a living day 8 embryo with Nomarski interference contrast optics. Two planes of focus are shown; $A$ is dorsal to $B$. Cells and fiber bundles are labeled as in Figure 5. The more dorsal of the two MP3 progeny, indicated by the solid arrow, transforms into the $\mathrm{H}$ cell.

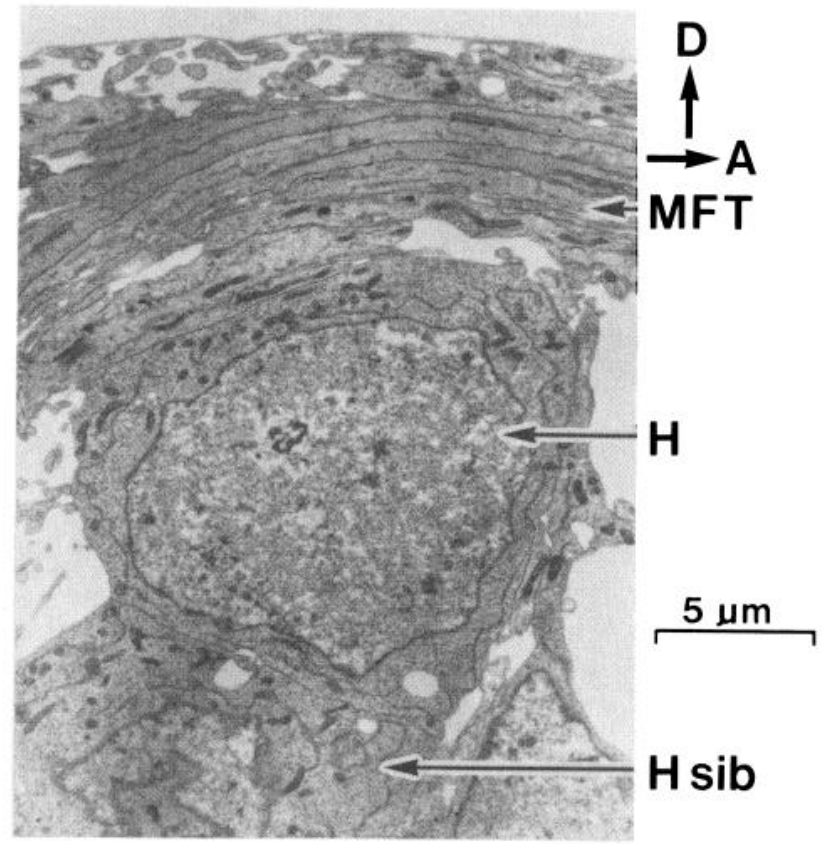

Figure 7. Electron micrograph of a sagittal midline section through the metathoracic segment of a day 8 grasshopper embryo. $D$, dorsal; $A$, anterior. One of the two somata of the MP3 progeny is dorsal to the other; this cell inevitably becomes the $\mathrm{H}$ cell. The more dorsal soma is contacted by the median fiber tract (MFT). At this stage, the MFT contains the processes of the MP4 progeny and the early progeny of the MNB. Magnification is $\times \quad 6000$.

terminal abdominal ganglion. Whereas the original single contralateral process of each of the MP3 progeny is in the median portion of the enlarging longitudinal fiber tracts, the new processes of the transforming cell are added laterally (Figs. 8 and $9 A$ ).
The original unilateral process disappears between days 11 and 12 and the transformed cell fully differentiates into the unpaired and bilaterally symmetric identified " $H$ " neuron (Figs 8 and $9 B$ ) (called the " $H$ " cell because of its distinctive morphological appearance). After day 11, the $\mathrm{H}$ cell continues to undergo two morphological changes: its cell body enlarges to a diameter of over $40 \mu \mathrm{m}$ and its symmetrical central arborizations rapidly expand (Fig. 8).

During its transformation, the new axon of the $\mathrm{H}$ cell initially follows the dorsal median fiber tract, the pathway first established by the MP4 progeny about day 6 . By day 8 , when the transformation begins, the median fiber tract has enlarged with the addition of the axons of the early progeny of the MNB (Figs. $6 \mathrm{~A}$ and 7). On this same day in which the transformation begins, the early progeny of the MNB are highly electrically coupled to the two MP3 progeny; one likely site for this coupling is where their axons, in the dorsal median fiber tract, extend directly over the more dorsal of the two somata of the MP3 progeny. During its transformation, the $\mathrm{H}$ cell comes to acquire many of the same morphological features as the first progeny of the MNB.

Examination of embryos on days 8 to 10 shows that either one of the two MP3 progeny can undergo the transformation, and thus on day 11, the partially transformed $\mathrm{H}$ cell can have its original unilateral process in either the left (Fig. 9A) or right (Fig. 8) longitudinal fiber tract. Of the $13 \mathrm{H}$ cells filled with dye in day 11 embryos, six had single processes in the left and seven in the right LFTs.

During the transformation of the $\mathrm{H}$ cell between days 8 and 12, the somata of the two MP3 progeny migrate dorsally to assume a new position. The soma of the $\mathrm{H}$ cell initially lies immediately ventral to the dorsal median fiber tract, at the very center of the early central fiber 


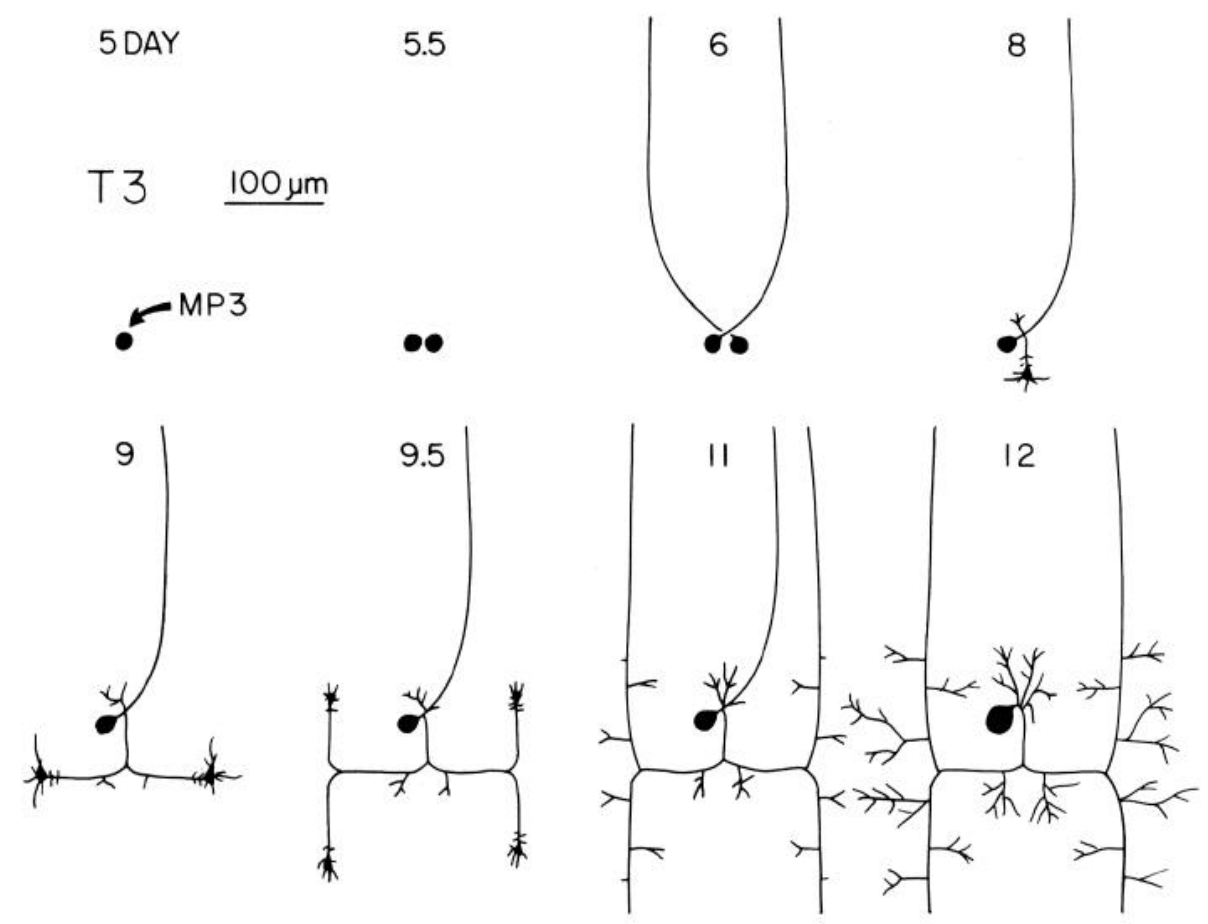

Figure 8. The transformation of the $\mathrm{H}$ cell in the metathoracic segment between day 5 and day 12 of embryogenesis. These are camera lucida drawings from cells injected with the fluorescent dye Lucifer Yellow.
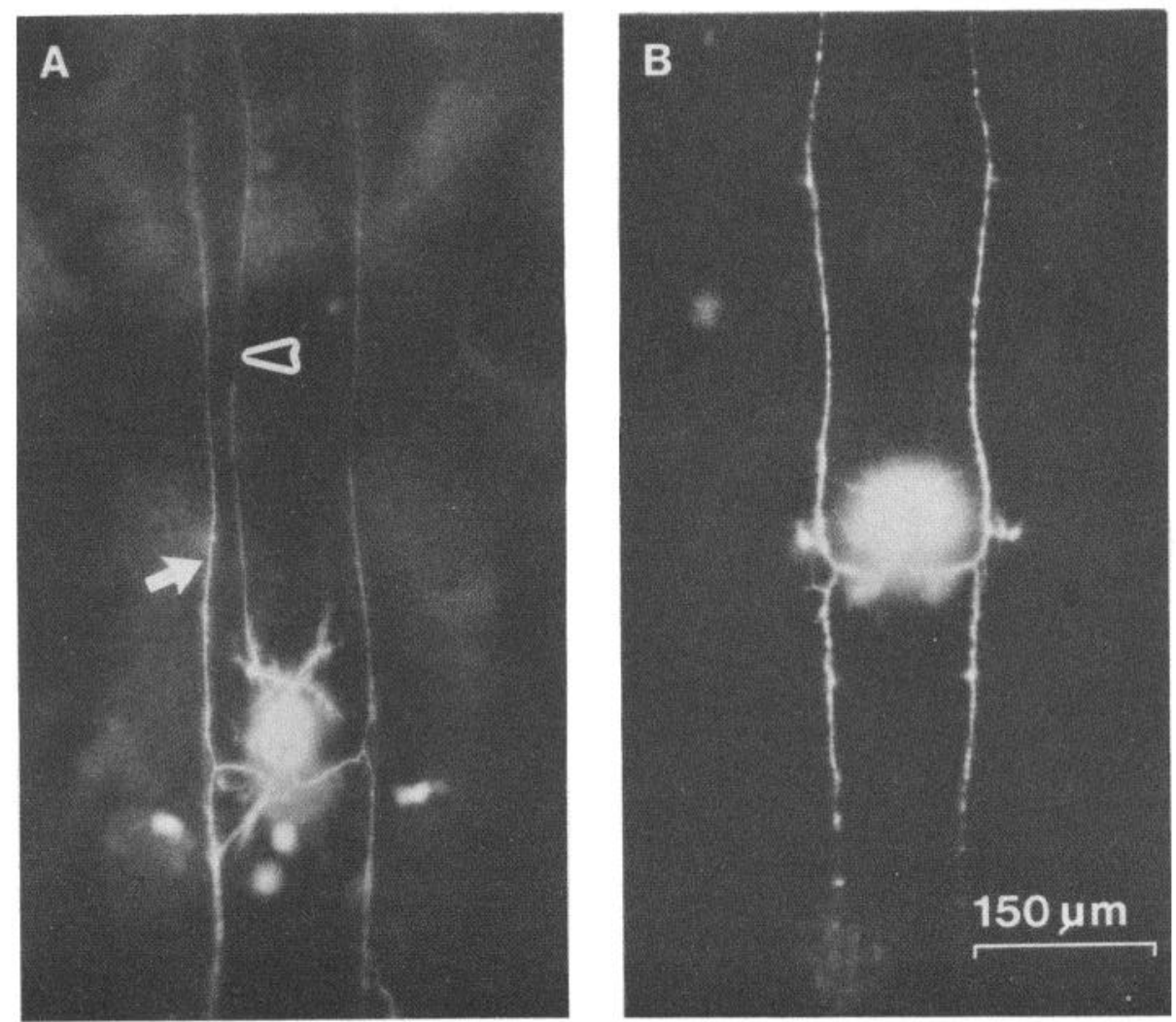

Figure 9. Photomicrographs of the $\mathrm{H}$ cell filled with Lucifer Yellow at two different stages of its transformation. $A$, On day 11, the original process is still present (caret) while the new processes have already extended (open arrow). B, On day 12 , the original process has disappeared and the cell body has begun to enlarge. In both cases, the cells appear larger than they are due to the intense fluorescence. The small fluorescent objects, particularly in $A$, are yolk and not dye. 
tracts. This initial position is quite separate from and anterior to the progeny of the MNB (Figs. 5 and 6). The somata of the MP3 progeny move around the median fiber tract and migrate dorsally. As the somata of the MNB progeny extend anteriorly and dorsally over the posterior commissure, the MP3 progeny assume a position immediately anterior to (and often just ventral to) the oldest progeny of the MNB (Fig. 10). The $\mathrm{H}$ cell and $\mathrm{H}$ cell sib often lie just outside the glial sheath that encases the somata of the progeny of the MNB; in some embryos, however, they appear to become incorporated within the glial sheath after day 11 .

\section{Physiological transformation of the $\mathrm{H}$ cell in the $\mathrm{T} 3$ segment}

The $\mathrm{H}$ cell acquires many of the same physiological properties as the first three progeny of the MNB in the T3 segment (Goodman and Spitzer, 1979; Goodman et al., 1979b; Goodman and Spitzer, 1980a, b). In day 18 embryos, the $\mathrm{H}$ cell is sensitive to iontophoretic application of acetylcholine (ACh) and $\gamma$-aminobutyric acid (GABA); the ionic dependence, reversal potential, and pharmacology of the responses to ACh and GABA are similar to those previously described for the MNB progeny (Goodman and Spitzer, 1979). The $\mathrm{H}$ cell receives spontaneous synaptic input (excitatory and inhibitory). The $\mathrm{H}$ cell is electrically uncoupled and electrically excitable in day 18 embryos (Fig. 11). The soma has a resting potential of $-60 \mathrm{mV}$ and an input resistance of 250 to 400 megohms. Action potentials in the $\mathrm{H}$ cell can be elicited by intracellular stimulation of the soma (Fig. 11) or extracellular stimulation of one of the ventral nerve cords containing its axons (not illustrated). The overshooting action potential in the soma has been shown to depend on an influx of both $\mathrm{Na}^{+}$and $\mathrm{Ca}^{2+}$ and the action potentials in the axons depend primarily on $\mathrm{Na}^{+}$ (Goodman and Spitzer, 1980a); this is similar to the first three progeny of the MNB (DUM 3, 4, 5; DUM 4, 5; and DUM 5).

In contrast to the $\mathrm{H}$ cell, its sibling (in embryos in which it survives past day 11) only develops the ability to make action potentials in its axon (Fig. 11). These $\mathrm{Na}^{+}$-dependent action potentials in the axon are passively propagated into the soma and thus do not overshoot the zero potential. When outward current is blocked by tetraethylammonium (TEA), the $\mathrm{H}$ cell ac-

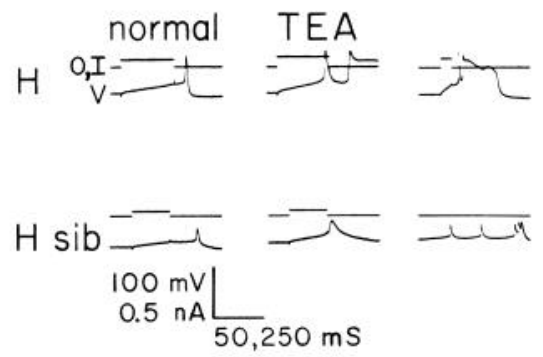

Figure 11. Electrical excitability of the $\mathrm{H}$ cell and $\mathrm{H}$ cell sib in the metathoracic ganglion of a day 18 embryo. The overshooting action potential of the $\mathrm{H}$ cell in normal saline is prolonged by addition of $30 \mathrm{~mm}$ tetraethylammonium (TEA). The $\mathrm{H}$ cell sib produces a small action potential that arises in the process. TEA causes only a slight broadening of this spike whether elicited by a depolarizing pulse or by steady depolarization. The records are from two cells.
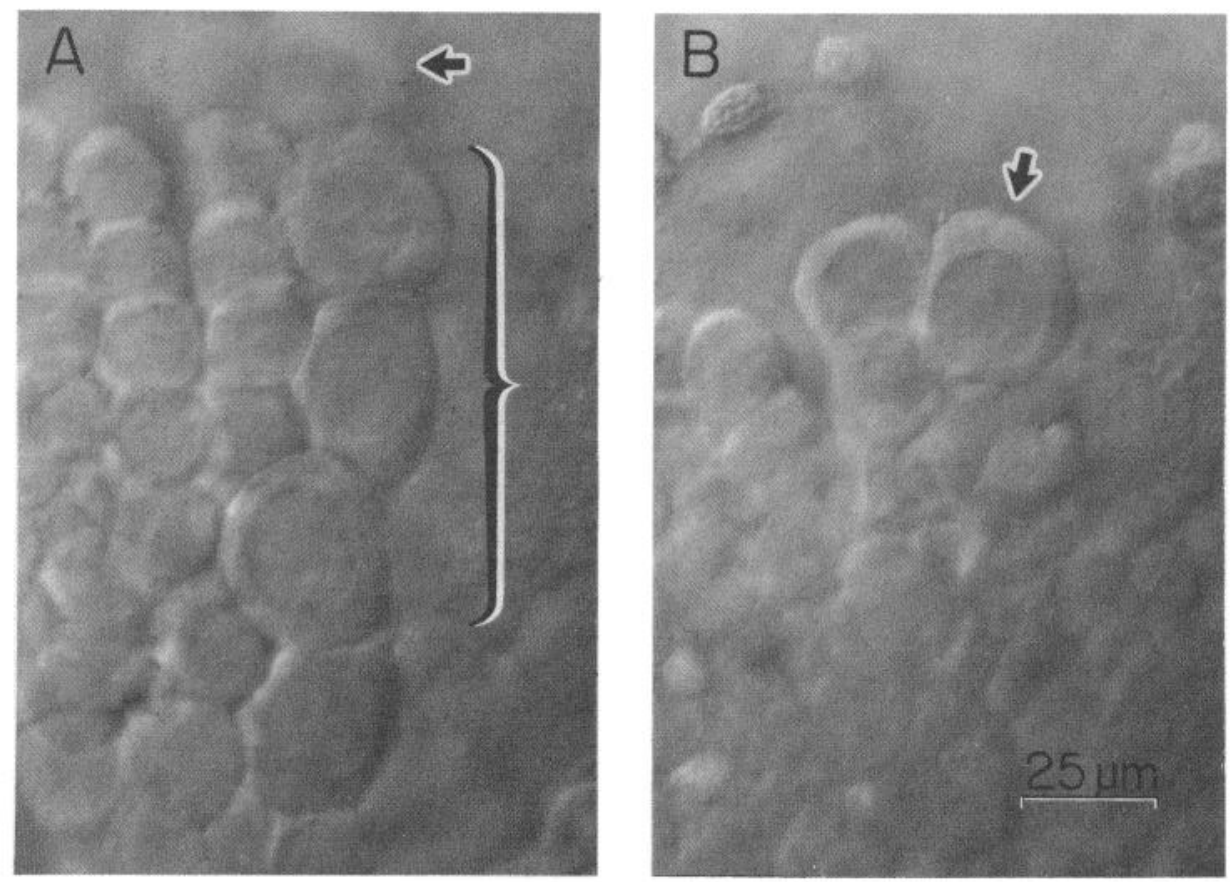

Figure 10. Photomicrographs of the dorsal surface of the metathoracic ganglion of a day 18 embryo visualized with Nomarski interference contrast optics. The sheath over the dorsal surface has been removed, as well as the glial sheath around the somata of the MNB progeny. The plane of focus in $A$ is dorsal to that in $B$. A, The three oldest progeny of the MNB (from top to bottom, DUM 3, 4, 5; DUM 4, 5; and DUM 5) are indicated by the bracket. The MP3 progeny lie deep to the focal plane (arrow) and anterior to the MNB progeny. B, The two progeny of MP3. Arrow indicates the H cell. 
tion potential broadens and assumes a characteristic wave form: an initial spike(s) dependent predominantly on $\mathrm{Na}^{+}$, followed by a plateau dependent chiefly on $\mathrm{Ca}^{2+}$ (Fig. 11) (Goodman and Spitzer, 1980a). In contrast, the action potential of the $\mathrm{H}$ cell sib broadens, but does not overshoot the zero potential and shows no plateau. Thus, we find no evidence for inward current channels in the soma of the $\mathrm{H}$ cell sib.

The two MP3 progeny are highly electrically coupled on day 6. On day 8, one of the two cells begins to transform while the two cells are still highly electrically coupled (coupling coefficient, $J>0.7$ ) and dye coupled. The morphological transformation of the $\mathrm{H}$ cell between days 8 and 12 takes place while it remains electrically coupled to its sib (Fig. 12). On day 12, overshooting action potentials can be recorded in the $\mathrm{H}$ cell which spread electrotonically into its sib; the action potentials can be prolonged by addition of TEA, providing a clearer demonstration of the coupling. By day 13, however, the $H$ cell and its sibling are electrically uncoupled $(\mathrm{d}<$ 0.001 ); neither action potentials nor steady polarization of the membrane of the $\mathrm{H}$ cell produces any detectable change in the membrane potential of the $\mathrm{H}$ cell sib. The $\mathrm{H}$ cell becomes excitable between days 11 and 13 (Good-

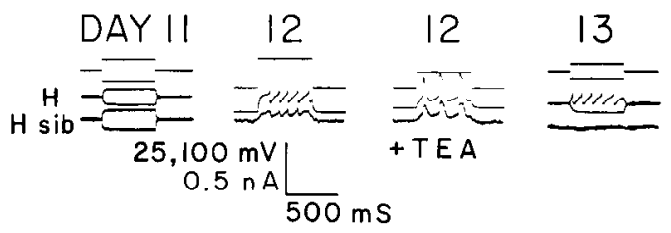

Figure 12. Electrical coupling of the two MP3 progeny: the $\mathrm{H}$ cell and the $\mathrm{H}$ cell sib. The two cells are electrically coupled and inexcitable on day 11 . They are coupled and the $H$ cell is excitable on day 12 as shown in normal saline and in TEA. The cells are uncoupled on day 13 . The records are from three pairs of cells. man and Spitzer, 1980b). Action potentials can first be generated in the new axons between days 11 and 12 and shortly thereafter in the soma.

In those embryos in which the $\mathrm{H}$ cell sib dies between days 10 and 12 , it never becomes excitable. It is only in those cases in which the $\mathrm{H}$ cell sib persists that it acquires the ability about day 12 to make action potentials in its axons but not its soma.

\section{Discussion}

We have described the origin and transformation of a single cell which assumes two different roles during grasshopper embryogenesis. It first appears as one of a pair of central pioneer fibers and then transforms into an unpaired identified neuron. The seven midline precursors (MPs) give rise to 14 progeny whose processes are either the first or are among the earliest processes to establish the initial axon pathways in the CNS. The two MP3 progeny each have a single process which decussates in the anterior commissure and extends anteriorly as one of the first fibers in the longitudinal fiber tracts. In many of the abdominal ganglia, the MP3 progeny die during later embryogenesis (described in detail in Bate et al., 1981) and never become excitable. These results support the notion that the processes of the MP progeny do not serve to carry electrical signals but rather have another role in the developing nervous system. Their role is likely to be as central guide fibers for later neurons whose axons project over the same route. Many of the other MP progeny also appear destined to die after they have served their pioneering role.

The MP progeny thus appear to be important structural elements for the organization of the early developing nervous system. This role may initially have been a temporary one. We suggest that in the course of evolution, one of the two MP3 progeny in the T3 segment became stabilized and passes through a second phase of

MNB progeny
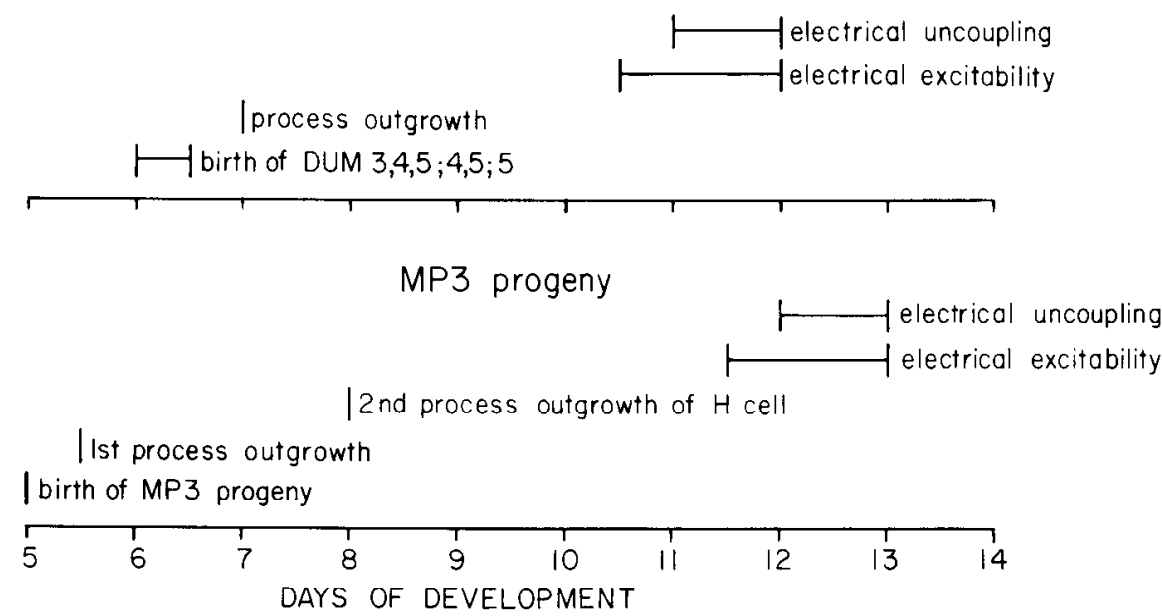

Figure 13. Temporal sequence of the development of the first three progeny of the median neuroblast (MNB) and the two progeny of midline precursor 3 (MP3) in the metathoracic segment between days 5 and 14 of grasshopper embryogenesis. The time interval between the final process outgrowth and the development of electrical excitability and electrical uncoupling is the same for both sets of neurons. 
differentiation. The $\mathrm{H}$ cell is temporally a dual-function cell, beginning its life as a typical MP descendant and later transforming into a neuron with properties like those of the progeny of neuroblasts (NBs). In particular, the $\mathrm{H}$ cell acquires many of the same morphological and physiological properties as the first three progeny of the MNB: DUM 3, 4, 5; DUM 4, 5; and DUM 5. The axons of the first MNB progeny extend in the median fiber tract and contact the more dorsal soma of the two MP3 progeny before day 8 (Fig. 7); the MNB progeny and MP3 progeny are electrically coupled on day 8, presumably at this location. The more dorsal soma of the two MP3 progeny transforms into the $\mathrm{H}$ cell. The morphological transformation of the $\mathrm{H}$ cell begins on day 8 by the extension of a new growth cone which runs posteriorly in the same median fiber tract (Figs. 6 and 8). Eventually the $\mathrm{H}$ cell grows a whole new set of axons, loses its original process, and becomes an unpaired, bilaterally symmetrical neuron whose electrical properties and other phenotypes are similar to DUM 3, 4, 5; DUM 4, 5; and DUM 5. The $\mathrm{H}$ cell soma comes to assume a position immediately anterior to the somata to these first three MNB progeny (Fig. 10).

During this transformation, the $\mathrm{H}$ cell not only acquires many of the same properties as the first three progeny of the MNB, but it acquires them in the same temporal sequence (Fig. 13). The relative timing of the outgrowth of processes, the cessation of electrical coupling, and the onset of electrical excitability (Goodman and Spitzer, 1980b) are similar for the first three MNB progeny and the $\mathrm{H}$ cell. The entire sequence of these phenotypes is simply delayed by about 1 day for the $\mathrm{H}$ cell beginning with the outgrowth of its new processes.

We do not know yet the relative contributions of the mitotic ancestry and the cellular environment in determining the transformation of the $\mathrm{H}$ cell in the T3 segment. However, we make the following observations. ( $i$ ) The MP'3 produces two progeny, one on the left and one on the right of the midline. The position of each cell allows us to predict in which longitudinal fiber tract its process will run. The cells are placed in this position by a fixed lineage, and thus we cannot say whether it is the position of the cell or its ancestry which dictates in which longitudinal fiber tract its initial process will run. (ii) One of the two cells transforms into the $H$ cell. Its decision to do so is independent of its left/right position. (iii) One of the two cells usually lies more dorsal than the other, and this cell inevitably becomes the $\mathrm{H}$ cell. Thus, the decisive factor underlying which cell transforms appears to be its dorsal/ventral position, and this may be independent of its ancestry.

Are there environmental factors which could selectively affect the more dorsal of the two cells? The soma of the more dorsal cell is contacted by the processes of the MP4 progeny and then by the axons for the first MNB progeny before and during its transformation (Figs. 6 and 7). The transforming $\mathrm{H}$ cell then comes to acquire many of the same phenotypes as the first MNB progeny. These observations lead us to suggest that the transformation may be an epigenetic event induced by cellular interactions with the axons of the MNB progeny, although influences from other neighboring cells may also occur. This hypothesis implies that a cell which has already undergone its initial differentiation can, in certain cellular environments, be induced to undergo a second phase of differentiation in both its morphology and physiology. This hypothesis is further supported by the observations of segment-specific differences in the transformation of the $\mathrm{H}$ cell, as described in the following paper (Bate et al., 1981).

\section{References}

Bate, C. M. (1976a) Embryogenesis of an insect nervous system. I. A map of the thoracic and abdominal neuroblasts in $L o$. custa migratoria. J. Embryol. Exp. Morphol. 35: 107-123.

Bate, C. M. (1976b) Pioneer neurons in an insect embryo. Nature 260: 54-56.

Bate, C. M. (1978) Development of sensory systems in arthropods. In Handbook of Sensory Physiology: Development of Sensory Systems, M. Jacobson, ed., Springer-Verlag, Berlin.

Bate, C. M., and E. B. Grunewald (1980) Embryogenesis of an insect nervous system. II. A second class of neuronal precursor cells and the origin of the intersegmental connectives. J. Embryol. Exp. Morphol., in press.

Bate, M., C. S. Goodman, and N. C. Spitzer (1981) Embryonic development of identified neurons: Segment-specific differences in the $\mathrm{H}$ cell homologues. J. Neurosci. 1 : 103-106.

Bentley, D., H. Keshishian, M. Shankland, and A. ToroianRaymond (1979) Quantitative staging of embryonic development of the grasshopper, Schistocerca nitens. J. Embryol. Exp. Morphol, 54: 47-74.

Edwards, J. S., M. W. Berns, and S. Chen (1979) Laser lesions of embryonic cricket cerci disrupt guidepath role of pioneer fibres. Soc. Neurosci. Abstr. 5: 158.

Goodman, C. S., and N. C. Spitzer (1979) Embryonic development of identified neurons: Differentiation from neuroblast to neuron. Nature 280: 208-214.

Goodman, C.S., and N. C. Spitzer (1980a) The mature electrical properties of identified neurons in grasshopper embryos. J. Physiol. (Lond.), in press.

Goodman, C. S., and N. C. Spitzer (1980b) The development of electrical properties of identified neurons in grasshopper embryos. J. Physiol. (Lond.), in press.

Goodman, C. S., M. Bate, and N. C. Spitzer (1979a) Origin, transformation, and death of neurons from an identified precursor during grasshopper embryogenesis. Soc. Neurosci. Abstr. 5: 161.

Goodman, C. S., M. O'Shea, R. McCaman, and N. C. Spitzer (1979b) Embryonic development of identified neurons: Temporal pattern of morphological and biochemical differentiation. Science 204: 219-222.

Goodman, C. S., K. G. Pearson, and N. C. Spitzer (1980) Electrical excitability: A spectrum of properties in the progeny of a single embryonic neuroblast. Proc. Natl. Acad. Sci. U. S. A. 77: $1676-1680$.

Keshishian, H. (1980) The origin and morphogenesis of pioneer neurons in the grasshopper metathoracic leg. Dev. Biol., in press.

Patterson, P. (1978) Environmental determination of autonomic neurotransmitter functions. Annu. Rev. Neurosci. 1: 1-17.

Spitzer, N. C., M. Bate, and C. S. Goodman (1979) Physiological development and segmental differences of neurons from an identified precursor during grasshopper embryogenesis. Soc. Neurosci. Abstr. 5: 181.

Truman, J. W., and S. E. Reiss (1979) Dendritic reorganization of an identified motoneuron during metamorphosis of the tobacco hornworm moth. Science 192: 477-479.

Wheeler, W. M. (1893) A contribution to insect embryology. J. Morphol. 8: 1-161.

White, J. G., D. G. Albertson, and M. A. R. Anness (1978) Connectivity changes in a class of motoneurone during the development of a nematode. Nature 271: 764-766. 